\title{
Dental Implants in Medically Compromised Patient- A Review
}

Dr. Inayaat Virk ${ }^{1 *}$, Dr. Shilpa Sunil Khanna M.D.S ${ }^{2}$, Dr. Rahul Vinay Chandra Tiwari, FOGS, MDS ${ }^{3}$, Dr. Munshi M.A.I $\mathrm{MDS}^{4}$, Dr. Heena Tiwari, BDS ${ }^{5}$, Dr. Rishabh Bhanot ${ }^{6}$

${ }^{1}$ Consultant Dental Surgeon, Indus Hygiea, SCF 21, Phase 6, Mohali, Punjab, India

${ }^{2}$ Department of Oral and Maxillofacial Surgery, Senior Lecturer, Sri Ramakrishna Dental College and Hospital, Coimbatore 641006, Tamil Nadu, India

${ }^{3}$ Consultant Oral \& Maxillofacial Surgeon, CLOVE Dental \& OMNI Hospitals, Visakhapatnam, Andhra Pradesh, India

${ }^{4}$ Oral and Maxillofacial Surgery, Reader, Sri Ramakrishna Dental Colllege and Hospital, Coimbatore, Tamil Nadu, India

${ }^{5}$ PGDHHM, Government Dental Surgeon, Chhattisgarh, India

${ }^{6}$ Consultant Oral and Maxillofacial Surgeon, Jyoti Kendra General Hospital, Ludhiana

DOI: $10.36348 / \mathrm{sjm} .2020 . \mathrm{v} 05 \mathrm{i} 02.002$

| Received: 14.01.2020 | Accepted: 29.01.2020 | Published: 07.02.2020

*Corresponding author: Dr. Inayaat Virk

Abstract

Systemic diseases hold major importance in relation to the prosthetic restorations supported by the dental implants in medically compromised patients. It is essential to monitor each stage of the treatment from beginning of the treatment plan to the long term follow-up it is under thereby a necessity of the interdisciplinary approach to the underlying disorder. Keywords: dental implant, medically compromised patients, systemic diseases.

Copyright @ 2020: This is an open-access article distributed under the terms of the Creative Commons Attribution license which permits unrestricted use, distribution, and reproduction in any medium for non-commercial use (NonCommercial, or CC-BY-NC) provided the original author and source are credited.

\section{INTRODUCTION}

A medically compromised patient can be benefited from dental implant rehabilitation, which in return will increase the quality of life of the patient to a significant level [1]. In order to give dental implant therapy in medically compromised patients, the practitioner should keep in mind that there is an altered general reactivity of the body and also decreased immunity to fight infections. These factors predispose the patient to various complications during and immediately after the surgery or on a long term duration surgery. Thereby, it is of great importance to take precautions in such patients not only during and after the surgery but also in the prosthetic phase as well as during the monitoring phase [2].

\section{Dental Implants and Medically Compromised Patients}

Because of the inter-relationships between body and the oral cavity, till the time patient presents for the treatment of dental implants, the systemic disease would already have had repercussions in the oral cavity. It is a known fact that many general sickness stand out particularly in the oral cavity, like diabetic patients have a more aggressiveperiodontal as compared to non-diabetic patients [3]. There are number of side effects of the medications the patient receives for his systemic chronic diseases [4]. These drugs have many consequences both in the oral cavity as well as to the whole body such as altered hematopoiesis (barbiturates), abnormal bleeding (nonsteroidal drugs antiinflammatory), decreased stress tolerance (beta-blockers), altered host resistance (insulin, antibiotics), gingival hyperplasia (nifedipine), xerostomia (many drugs), respiratory depression (narcotics).Hence, these factors condition and limit a number of therapeutic measures, mostly in the surgical stage of dental implant therapy [5]. The systemic diseases can lead to accidents and intraoperative complications, for example; a patient with heart disease can go into cardiac depression during implant surgery while endangering the vital functions [6]. Dental implant therapy in medically compromised patients should be designed in such a way that long-term complications should be taken into account, as these complications are more frequent as well as severe than in healthy patients, for example in case of a patient with osteoporosis, the time for osteointegration of the implant can increase as there is a bone resorption as the characteristic of this disease as well as there can be need for a specific manner for prosthetic loading, designed in such a way that it can stimulate bone healing [5, 6]. The most common types of systemic diseases are: cardiovascular disease: hypertension, atherosclerosis, valvular heart diseases, angina pectoris; hematologic disorders: anemia, leukemia; endocrine disorders: diabetes, thyroid disease, adrenal gland disorders lung: emphysema, chronic bronchitis; liver disease: cirrhosis; disease of bone: osteoporosis, hyperparathyroidism. The elder patients also fall under the category of healthy compromised patients because 
of the age related physiological changes as well as they present with multiple associated diseases. There are changes in the local as well as general medical conditions in patients who smoke, thereby preventive measures and possible complications should be taken into account. In order to accomplish a successful surgical and prosthetic phases in these patients, there should be an appropriate treatment plan design by completely evaluating the general health and associated diseases. Antibiotic prophylaxis is one of the most important aspects in the treatment of medically compromised patients with dental implants. It is mandatory in patients with an increased risk of bacterial endocarditis, myocardial infarction, those with an immune suppression by corticosteroids or radiation therapy or diabetes [6]. In addition, the antibiotic prophylaxis is recommended in patients with anemia or liver disorders. Clinical laboratory tests are also very important in the whole treatment of these patients. The most commonly investigated clinical laboratory parameters are the following: complete blood cell count (CBC), serum glucose, bleeding tests, serum calcium, bilirubin, creatinine etc [7]. The key factor in the treatment planning and surgery procedures in medically compromised patients is the stress reduction protocol.

1. Patients with cardiovascular diseases are mostly on hypotensive medication that has the side effect of xerostomia, fungal infections of the mouth (candidiasis). Also, calcium blockers also produce gingival hyperplasia and erythema, both in natural dentition as well as around the dental implant. Due to the risk of intraoperative episode of angina pectoris in patients with cardiovascular diseases, there should be short treatment sessions and also the vasoconstrictor substances in anesthesia should be cautiously used. Moreover, bacterial endocarditis with very serious consequences can occur in patients with heart diseases. Hence the surgery should be as less aggressive as possible. Antibiotic prophylaxis of bacterial endocarditis is necessary in patients with high risk heart diseases. The patients who are on anticoagulants demand surgery procedures, which are less invasive, for example the flapless insertion of the dental implant. The bleeding is reduced to a minimum and the associated risks are reduced. The current concept in such cases is to continue the anticoagulant medication because of the risks of a myocardial infarction or stroke is more important than the intraoperative bleeding. Although, prothrombin time test should be done prior to surgery.

2. Patients with endocrine disorders, for example diabetes which is one of the most common diseases generally encountered in dental practice. Diabetes has several effects in the oral cavity like increased risk of inflammation and infection of gingival, aggressive periodontal disease, alveolar bone loss, candidiasis. In case of surgical approach in diabetic patients, stress reduction protocol should be employed in order to prevent the intraoperative hyperglycemia crisis.

3. It is of great importance to know that the insertion of dental implants can trigger a congestive heart failure, or may initiate a cardio-respiratory depression in patients who suffer from hyper or hypo thyroid disorders. The patients with chronic adrenal insufficiency like Addison diabetes may be at the same risk during surgical procedures. The Cushing's syndrome (hypofunction of the adrenal glands) has the consequences on both the condition of the patient (hypertension) and as well as delayed healing, with risk of secondary infection. Moreover, long-term corticosteroid therapy can also affect the dental implant. Corticosteroids are beneficial in reducing inflammation and pain as immediate postoperative effects, but corticosteroids delay the healing, decrease the blood leukocyte count and hence decrease the patient's ability of antibacterial defense.

4. In dental implantology, blood disorders are of the most critical diseases. There are both short term as well as long term complications in anemia like decreased bone density, delayed healing, and increased healing time. There are high chances of intraoperative bleeding in these patients which can lead to increased discomfort and postoperative edema. Also, it is associated with a high risk of secondary infections. There is low long-term implant survival because of frequent chronic infections. The success of the implants are highly compromised in case of leukocyte due to risk of multiple complications, among which the most common is infection which can occur during any stage of treatment. The intraoperative bleeding is high, and thereby the risk of postoperative edema and secondary infection are increased same as in case of anemic patients.

5. The medications taken by patients suffering from the chronic pulmonary diseases mostly are corticosteroids which cause suppression of immune function of the body and as a result the response is altered in response to surgical stress. The possible complications have already been mentioned.

6. The process of hemostasis is affected by the liver diseases which interfere with surgical procedures and as a result of which the intraoperative bleeding is increased in such patients. There can also be a respiratory depression because of the low capacity to metabolize medicinal substances.

7. Bone resoprtion is mostly attributed to the skeletal disorders. The osteoporosis involving the jaw bone where the implant is desired to be inserted will have the same features as the other bones of the body such as thinning of cortical bone, demineralization and decrease of trabecular pattern. The medication used for osteoporosis is another important aspect, i.e; bisphosphonates 
(BPs) and osteonecrosis of the jaw is a complication observed in patients who are on oral or intravenous bisphosphonates, which is called as bisphosphonate-related osteonecrosis of the jaw (BRONJ). It is very crucial to inform all the patients who are undergoing bisphosphonate therapy about the possible risks of the development of osteonecrosis. All such patients who are about to undergo implant surgery should be enquired about bisphosphonate therapy including the dosage, and length of treatment before the surgery since all of these factors influence the treatment protocol. Hyperparathyroidism affects the jaws is similar way as osteoporosis such as altered trabecular bone pattern, loss of lamina dura etc. The effects on dental implants are the same with that with osteoporosis.

Patients with age over 60 years old have a lesser physiological adaptability to stress and hence they should be treated similar to the patients with mild general medical conditions. Therebby, it requires a greater caution both intra-and post-operatively. In order to determine the therapeutic approach for dental implants, systemic disorders are to be well taken into account. The routine medications that are taken by the elderly people interfere with the status of oral cavity status by the side effects [7]. Smoking is an adverse habit with local as well as systemic complications; hence special measures should be taken because of the decreased resistance to inflammation of the gums and tissues around the implant, delayed healing of the soft tissue and decreased resistance to infection after any oral surgery in smokers [8]. Furthermore, the systemic diseases and poor health status of the patient affect the preoperative as well as the surgical and prosthetic phases. According to the systemic disease, special precautions that need to be taken in medically compromised patients are: the operative protocol of as minimum stress as possible should be followed by avoiding major interventions with a long duration of time for the prevention of intraoperative complications such as cardiovascular-respiratory depression or angina attack, collagen sponges or special type of sutures should be used in order to achieve hemostasis, antibiotic prophylaxis and antibiotics therapy should be given to decrease the risk of secondary infection [9].

\section{CONCLUSIONS}

In order to avoid failures in medically compromised patients who receive dental implant therapy, it is obligatory to know the implications of systemic diseases as well as those produced by the current medications in the oral cavity. By keeping in mind the altered biological constants of the patient, the medical team must be able to solve the complications that occur and should also be prepared with more treatment options for that situation.

\section{REFERENCES}

1. Bornstein, M. M., Cionca, N., \& Mombelli, A. (2009). Systemic conditions and treatments as risks for implant therapy. Int J Oral Maxillofac Implants, 24(Suppl), 12-27.

2. Courtney Jr, M. W., Snider, T. N., \& Cottrell, D. A. (2010). Dental implant placement in type II diabetics: a review of the literature. J Mass Dent Soc, 59(1), 12-14.

3. Javed, F., \& Romanos, G. E. (2009). Impact of diabetes mellitus and glycemic control on the osseointegration of dental implants: a systematic literature review. Journal of periodontology, 80(11), 1719-1730.

4. Van Steenberghe, D., Quirynen, M., Molly, L., \& Jacobs, R. (2003). Impact of systemic diseases and medication on osseointegration. Periodontology 2000, 33(1), 163-171.

5. Dereka, X., Mardas, N., Chin, S., Petrie, A., \& Donos, N. (2012). A systematic review on the association between genetic predisposition and dental implant biological complications. Clinical oral implants research, 23(7), 775-788.

6. Mombelli, A., \& Cionca, N. (2006). Systemic diseases affecting osseointegration therapy. Clinical oral implants research, 17(S2), 97-103.

7. Glösel, B., Kuchler, U., Watzek, G., \& Gruber, R. (2010). Review of dental implant rat research models simulating osteoporosis or diabetes. International Journal of Oral \& Maxillofacial Implants, 25(3):516-524.

8. Schou, S., Holmstrup, P., Worthington, H. V., \& Esposito, M. (2006). Outcome of implant therapy in patients with previous tooth loss due to periodontitis. Clinical oral implants research, 17(S2), 104-123.

9. Wilson, V. (2013). An insight into periimplantitis: a systematic literature review. Primary dental journal, 2(2), 69-73. 